\title{
Geochemical constraints on bacteriophage infectivity
}

\author{
HANS K CARLSON ${ }^{1}$, DENISH PIYA ${ }^{2}$, MADELINE L \\ MOORE $^{1}$, ADAM M DEUTSCHBAUER $^{1}$, ADAM P ARKIN ${ }^{1}$ \\ AND VIVEK K MUTALIK ${ }^{1}$ \\ ${ }^{1}$ Lawrence Berkeley National Laboratory \\ ${ }^{2}$ UC Berkeley \\ Presenting Author: hkcarlson@lbl.gov
}

Bacteriophage are potent and selective inhibitors of microbial growth and are known to have profound impacts on microbiome composition and function. Inorganic ions are critical for phage infectivity, but relatively little quantitative data is available on the threshold concentrations of ions to enable phage predation aside from common alkali earth metal/alkali metals such as $\mathrm{Ca} 2+, \mathrm{Mg} 2+, \mathrm{Na}+, \mathrm{K}+$. We measured the half-maximal effective concentrations (EC50) of 80 different inorganic ions for the model infection of $E$. coli with T4 phage. We found that most alkali earth metals/alkali metals enable infection at concentrations between $1 \mathrm{mM}$ and $100 \mathrm{mM}$. However some ions such as $\mathrm{Cs}, \mathrm{Be}$, transition metals and reactive oxygen and nitrogen species (ROS/RNS) are toxic to E. coli at concentrations lower than $1 \mathrm{mM}$ and we find that in many cases the presence of phage protects bacteria from ion toxicity likely by sequestering these ions and scavenging RNS/ROS. We leverage our findings and geochemical field datasets to predict how variable environmental conditions influence phage infectivity and discuss implications for the ecology of phage and the magnitude of their impact on microbial processes in various environments. 